\title{
On Thermodynamic Aspects of the Efficient Power Engineering
}

\author{
Igor I. Samkhan
}

\author{
Yaroslavl State Technical University, Yaroslavl, Russia
}

\begin{abstract}
The possibility of using the statically non-equilibrium systems to increase the power cycle efficiency is investigated here. A method of dynamic equilibrium that was applied in this analysis allows the differences of energy potentials between a system and an environment as additional components of the equations of system state to be taken into account. In this model, a dependence of entropy not only on the thermal effects, as usually, but on the mechanical ones is considered too. Besides, the adiabatic processes running with entropy decreasing are feasible here as well. The consequence of such an approach is the opportunity of creating new regenerative cycles with high efficiency for both the powergenerating systems and the refrigeration ones. The closing of such cycles in this case can be carried out with the transformation of the consumed mechanical energy not only into the thermal energy, as usual, but also into the kinetic energy. The second law of thermodynamics does not put obstacles to extra improvement of the cycle efficiency since for the open systems the performance of a cycle can be determined by the relative difference of temperatures of the working fluid (but not necessarily by the temperatures of the thermal sources) at the upper and lower temperature levels of the cycle. The received results satisfy the general laws of conservation and completely coincide with the classical approach in the quasistatic approximation. They create the necessary prerequisites for an effective energy development.
\end{abstract}

Keywords: Thermodynamics, dynamic equilibrium method, equations of conservation and state, Bernoulli equation, first and second laws, regenerative cycles, efficiency.

\section{INTRODUCTION}

The modern analysis of the Carnot cycle for ideal gas which limits the efficiency of the thermal machines by a difference of temperatures of hot and cold thermal sources is based on the application of the quasi-static equilibrium method in which it is assumed that the intensive parameters of a system practically coincide with the similar parameters of an environment and that the process velocities are low, and the properties of working fluids remain constant.

A consequence of these assumptions is the seeming conclusion about the exclusive connection of one of the system parameters, namely the entropy, with the heat exchange processes, which does not quite agree with the known equivalence principle of thermal and mechanical energy.

Though these assumptions are justified in many practically important cases, nevertheless they limit the generality of the obtained results and the prognosis completeness of energy development prospects.

At the same time, it is of interest to estimate additionally the degree of the fulfillment of these assumptions in various thermodynamic cycles and also the possibility to increase efficiency of thermal machines (cycles) outside the above assumptions.

In order to investigate these problems we analyze some typical cycles of both the closed equilibrium thermodynamic systems and the open non-equilibrium ones with using a

\footnotetext{
*Address correspondence to this author at the Yaroslavl State Technical University, Moscow Prospect 88, Yaroslavl, Post/Zip Code 150023, 41700, Russia; Tel/Fax: 7 (4852) 446833;

E-mails: eneres1@gmail.com, eneres@rambler.ru
}

method of dynamic equilibrium offered by the author, in which such a coincidence between the intensive system parameters and the similar parameters of an environment is not necessary [1].

Essentially, such an approach can be regarded as an application of the Boltzmann's principle coming from the statistical thermodynamics according to which the nonequilibrium states of a system can be described as the equilibrium ones with due regard for its out-of-balance potential energy [2]. Besides, such a method of the thermodynamic analysis is similar to the D'Alembert-Lagrange principle which determines the ratio between the statics and dynamics in the theoretical mechanics.

Partially, these questions connected with the relationship of the static and dynamic models of thermodynamics were considered earlier in a number of papers [3-5], and they have also been discussed on a number of international conferences $[6,7]$.

In the present paper, the developed conception of thermodynamics as well a comparison of its basic notions with those of the known static method is discussed in greater details.

\section{THE EQUATIONS OF CONSERVATION AND STATE OF THE OPEN THERMODYNAMIC SYS- TEMS}

Let us consider such a conception on an example of an ideal gas steady flow being a statically non-equilibrium system.

Typically such systems are described from the quasistatic standpoint owing to the introduction in the implicit 
shape of the assumption about identity of the equations of the first law of thermodynamics for the closed systems

$\delta q=d u+p d v$,

and the open systems

$\delta q=d h+\delta l_{0}$.

This admission can be expressed by the equality

$\delta q=d u+p d v=d h+\delta l_{0}$,

which defines a change of both an internal energy $d u=c_{v} d T$ and enthalpy $d h=d(u+p v)=c_{p} d T$ of a system under influence of such effects as heat $\delta q=c_{n} d T$, work of expansion (deformation) $\delta l=p d v$, technical work $\delta l_{t}$ or available work

$\delta l_{0}=-v d p=\omega d \omega+\delta l_{t}$.

In this case, kinetic energy $\delta w=\omega d \omega$ of the gas flow having velocity $\omega$ is associated only with the equilibrium system parameters such as temperature $T$, pressure $p$, specific volume $v$ or density $\rho$, and gas specific thermal capacities $c_{p}, c_{v}$ or $c_{n}$ at $p=$ const, $v=$ const or $p v^{n}=$ const respectively, which determine the static system states.

However, such an approach connected with applying the identical methods of description to the non-equilibrium open systems and the closed equilibrium ones is not proved strictly enough

Therefore in this paper, the new equations represented in the forms of (5) and (6) are offered to describe the law of energy conservation as applied to the open thermodynamic systems

$\delta q=d u+p d v+v d \Delta p=d h+\omega d \omega$

$\delta q-\delta l=d u+d(p v)+v d \Delta p=c_{p} d T+v d \Delta p=d h+\omega d \omega$.

In these equations unlike Eq. (2), a possible difference of energy potentials $v d \Delta p$ between a system and an environment is considered, and the classical definition of the available work according to Eq. (4) is replaced by the new expression (7)

$-\delta l=-\delta l_{0}=d h+v d \Delta p=d h+\omega d \omega$.

In this case, along with the system parameters such as $T$, $p, v$ and others, these equations take into account the conditions of non-equilibrium interaction of the system with the environment in the form of kinetic energy $\delta w$ of the gas flow and pressure difference $\Delta p=p-p_{0}$ with the environment.

With using these equations, the non-equilibrium systems can be considered as the dynamically equilibrium ones since the available out-of-balance potentials of such systems are counterbalanced by the appropriate changes of their kinetic energy $v d \Delta p=-\omega d \omega$.

The equations (5) and (6) may be transformed to the known Clapeyron equation $R T=p v$ as well the Mayer one $\left(c_{p}-c_{v}\right)=R$ under conditions of $c_{n}=c_{p}$ and $p=p_{0}$ [5].

At the same time, the definition (7) of the available work essentially coincides with the known definition of the technical work of an ideal adiabatic process and, unlike the equation (4), includes a change of total gas enthalpy or otherwise a change of stagnation enthalpy of a flow $\Delta h^{*}=\Delta h+\int v d \Delta p=\Delta h+\int \omega d \omega$.

Besides, in doing so, the equations (5) and (6) for the open systems unlike the known equation (2) can not be transformed into Eq. (1) for the closed static systems if the peculiarities of the open systems such as a difference of potential energy or a change of mass between a system and an environment take place.

Moreover unlike the traditional approach, the equations (5) and (6) can be represented in the form of the Bernoulli differential equation without using any assumptions of the quasi-static method. Thus, when being applied to an isentropic process, with using the transformation

$$
(k-1) d h / k=d(p / \rho)=\left(d p / \rho-a^{2} d \rho / k \rho\right)
$$

and taking into account that sound speed $a$ is defined by the expression $a^{2}=\partial p / \partial \rho$, it is possible to find that

$d h=c_{p} d T=d p / \rho=v d p$.

Then, for an isentropic process, in which $d s=0$, Eqs. (5) to (6) can be represented in the form of the Bernoulli equation

$-d p / \rho=d \omega^{2} / 2+\delta l_{t}$.

From the standpoints developed here, Eq. (10) characterizes only the adiabatic gas flows, and consequently it can not be considered as the common equation of energy in the mechanical shape as it was supposed earlier.

At the same time, Eq. (6) as well as Eq. (2) agrees with the known dependence of the velocity of a gas flow on its cross-sectional area $f$, the squared speed of sound $a^{2}=\partial p / \partial \rho$ and the Mach number $M=\omega / a$.

So, at a constant pressure of an environment $p_{0}=$ const this equation can be represented in the form of Eq. (11)

$\delta q-\delta l=d h+\left[\omega^{2} /\left(M^{2}-1\right)\right] d f / f$,

in which the known correlation (12) takes place

$\left(M^{2}-1\right) d \omega / \omega=d f / f$.

Besides, in this case the enthalpy of static systems $h_{c}$ and the enthalpy $h$ of dynamic systems $h$ are described by different equations and, in general, do not coincide, contrary to what was supposed earlier: 
$d h_{c}=d(u+p v)=d u+p d v=c_{v} d T+R d T /(n-1)=$

$=c_{v}(n-k) d T /(n-1)=c_{n} d T$

$d h=d(u+p v)=\delta h_{c}+v d p=$

$=c_{n} d T+n R d T /(n-1)=c_{p} d T$,

The equality $(d u+p d v)=c_{p} d T$, which is commonly used to justify the assumption $d h_{c}=d h$, is fulfilled only under the condition that $n=0$.

\section{PECUliarities OF THE ADIABATIC PROC- ESSES OF THE OPEN THERMODYNAMIC SYS- TEMS}

\subsection{Description of the Flows with Application of the Ber- noulli Equation}

Usually, from the standpoint of the quasi-static method the isentropic gas flows are considered as the only possible ones in the ideal adiabatic conditions.

In this case the integral forms of the differential Bernoulli equations (10) or (15)

$-d p / \rho=d \omega^{2} / 2$,

are represented by the expressions

$$
\begin{aligned}
& \Delta(p / \rho)+\Delta \omega^{2} / 2+R \Delta T /(k-1)=0 \\
& \Delta(p / \rho)+\Delta \omega^{2} / 2+R \Delta T /(k-1)+l=0,
\end{aligned}
$$

provided that the gas flow properties are described by the Poisson equation for a quasi-static adiabatic process

$p v^{k}=$ const,

where the exponent of an adiabatic curve is equal to $k=c_{p} / c_{v}$.

However, according to the Bernoulli equation, other adiabatic flow regimes are possible as well, for example, the flow regimes in which the ratio between the density $\rho$ or the specific volume $v$ and pressure $p$ of a gas flow is expressed by the equation of polytropic processes

$p v^{n}=$ const

with the polytropic exponent $n= \pm \infty[8]$.

In this case, the expression (17) can be represented by the equations (20) and (21)

$$
\begin{aligned}
& \Delta(p / \rho)+\Delta \omega^{2} / 2+R \Delta T /(n-1)=0 \\
& {[n /(n-1)] \Delta(p / \rho)=-\Delta \omega^{2} / 2,}
\end{aligned}
$$

in which the polytropic exponent $n$ can be defined by the expressions

$$
\begin{aligned}
& c_{v} \Delta T+l=R \Delta T /(k-1)+l=R \Delta T /(n-1), \\
& c_{p} \Delta T+l=k R \Delta T /(k-1)+l=n R \Delta T /(n-1),
\end{aligned}
$$

$n=\frac{c_{p} \Delta T \pm l}{c_{p} \Delta T \pm l-R \Delta T}$.

These equations show that such an exponent can also depend not only on the thermal influences, as it is usually supposed, but on the mechanical ones $l$, too.

Besides, according to the Bernoulli equation the adiabatic isothermal processes are feasible theoretically as well. Thus, if a polytropic exponent in Eq. (24) tends to unit and the density of a gas is specified by the ratio (25)

$p v=$ const ,

then the integral form of Eq. (10) coincides with the definition of the work needed for compression of a gas in an isothermal quasi-static process from the pressure $p_{1}$ to the pressure $p_{2}$ :

$l=R T \ln \left(p_{2} / p_{1}\right)=-\Delta \omega^{2} / 2$.

In this case the ratio of this work to the temperature of the said dynamic process

$$
\Delta s=l / T=-R \ln \left(p_{2} / p_{1}\right)
$$

coincides with the similar ratio of the quantity of heat to be released by the gas during its compression in a quasi-static process

$\Delta s=q / T=-R \ln \left(p_{2} / p_{1}\right)$.

And the last ratio is usually used in the quasi-static method for the definition of the entropy as a function depending only on the thermal interactions.

The above opportunities to regulate the regimes of an adiabatic gas flow and to represent the entropy not only as a function of the thermal interactions but also the mechanical ones are not considered in the modern quasi-static method.

Moreover, in this case such opportunities are considered as impossible in principle since they do not agree with some notions of the quasi-static method that are mechanically extended from the area of statics to the area of dynamics as well.

In particular, it is usually assumed that the absence of an external heat exchange, i.e. provided that $q=0$, is sufficient to consider an ideal gas flow as an isentropic one owing to the known definition $\Delta s=q / T=0$.

Besides, in descriptions of the adiabatic gas flows two concepts of heat capacity are simultaneously used, and they are not quite coordinated among themselves.

According to one of them the heat capacity of such a flow is equal to zero $c=q / \Delta T=0$ owing to the absence of heat exchange processes.

While, on the other hand, such a heat capacity, namely the heat capacity of a gas in a quasi-static isobaric process $c_{p}=q / \Delta T=$ const, can also be used for expressing a tem- 
perature dependence of a gas on its kinetic energy even in the case when any heat exchange processes are absent.

Such an approach is usually expressed by the known equations

$c_{p} d T=-d \omega^{2} / 2$,

$T_{2}=T_{1}\left[1 \pm(k-1) \Delta \omega^{2} /\left(2 a^{2}\right)\right]$

which do not correspond completely to the modern practice, for instance with reference to the flows taking place in the blades of active gas turbines where such flows can remain almost isothermal even despite of significant changes in flow velocity.

\subsection{The Flow Analysis with Application of the Common Energy Equation}

In the general case the open adiabatic systems can also be described by using the energy equation (7) that can have a wider field of application in comparison with that of the Bernoulli equation.

In turn, the equation (7) can be represented in the form of the expression (31)

$$
-T d s=d p / \rho+d \omega^{2} / 2+\delta l=d h_{c}+d \omega^{2} / 2+\delta l,
$$

which assumes the existence of the ideal gas flows with variable entropy and coincides with the Bernoulli equation if the entropy of a gas flow is constant.

In the expression (31) one takes into account the possibility of representing the enthalpy in terms of the equations

$$
\begin{aligned}
& d h=d u+p d v+v d p=T[(d u+p d v) / T]+ \\
& +v d p=d h_{T}+d h_{c}=T d s+v d p \\
& d h=d(u+p v)=d\left(c_{p} T\right)=d h_{c}+d h_{T}= \\
& =c_{p} d T+T d c_{p}=v d p+T d s
\end{aligned}
$$

in which the entropy of the dynamic adiabatic processes is defined by Eq. (34) coinciding formally with the similar equation of the classical entropy in the quasi-static method

$$
d s=d c_{p}=(d u+p d v) / T .
$$

Besides, it is taken here that $d h_{c}=(\partial h / \partial T)_{c} d T$, $d h_{T}=(\partial h / \partial T)_{T} d c_{p}$, and $c_{p} d T=v d p$ accordingly to Eq. (9).

A special feature of these equations is that they do not point to any obligatory connection of the entropy with the processes of external or internal heat exchange and satisfy the known result $d c_{p}=d s$ obtained earlier in [3] due to a statistical analysis of molecular distributions.

Earlier, the equations similar to Eqs. (32) and (33) were known with reference to the flows having thermal contacts with the environment. It is shown here that they are applicable also to the adiabatic flows in which thermal interactions are absent.
The appearance of thermal summands in the equations of the adiabatic flows is connected with the possibility of mutual transformation of kinetic, thermal, and mechanical energy, what is absent in the quasi-static processes.

Let us analyze such polytropic processes by the example of the steady subsonic gas flows in narrowed heat-insulated channels (nozzles), the images of which are shown in Fig. (1). These flows have the identical initial parameters $T_{1}, p_{1}, v_{1}$. Besides, the flows 1-2, 1-3 and 1-5 have the same final pressure $p_{2}=p_{3}=p_{5}<p_{1}$ as well.

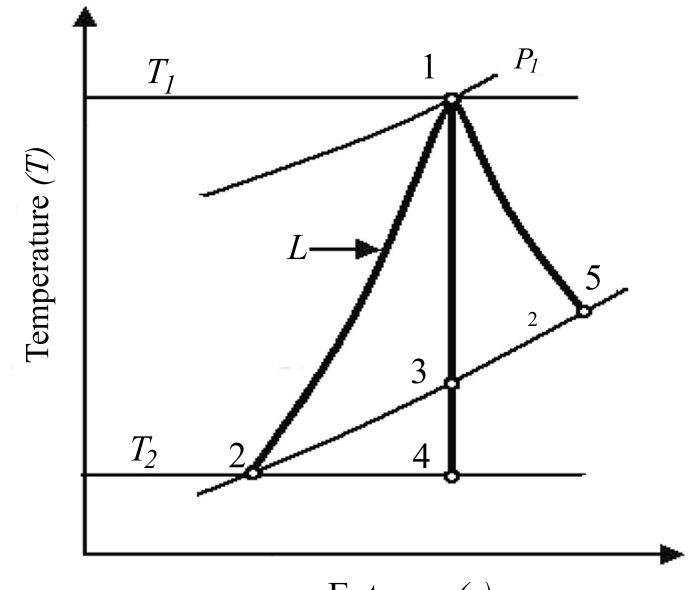

Entropy $(s)$

Fig. (1). The $T-s$ adiabatic curves of gas flows with an equal pressure difference and various conditions of mechanical interactions.

In the flow 1-2 a mechanic-geometrical nozzle is used in which the velocity of a gas flow can be increased additionally due to the gas obtaining the mechanical or potential energy with a simultaneous decrease in cross-section of the flow.

The flow 1-3 in this figure represents an isentropic expansion of a gas without any mechanical influences.

The flow 1-5 corresponds to the polytropic expansion of the gas in the same pressure interval, during which process the flow is obtaining additional heat from an internal energy sources, for example, at the expense of the heat arising due to frictional forces.

The flow 1-4 is an isentropic gas expansion without any mechanical effects from pressure $p_{1}$ to pressure $p_{4}<p_{2}$ provided that the gas final temperatures in the processes $1-2$ and 1-4 are the same $T_{2}=T_{4}$.

The adiabatic flows like the process 1-5 in which entropy can increase owing to the influence of internal thermal sources are well-known.

It is shown here that the dynamic adiabatic flows with decreasing entropy are possible too. At the same time, this notion does not relate to the adiabatic quasi-static processes in which lowering of entropy is not permitted in accordance with the second law of thermodynamics.

As is shown in Fig. (1), the flows 1-2, 1-3 and 1-5 have various final values of the temperatures, specific volumes and entropy changes, while their initial parameters and the 
pressure drop are the same; in doing so, these final parameters depend on the form and value of external influences.

Besides, under these conditions the mechanical energy being consumed by the flow in the process 1-2 results in additional lowering of the internal energy of the gas, its temperature and entropy in comparison with the similar parameters of the isentropic expansion of the same gas in the process 1-3 when any influences in the form of heat or work are absent.

At the same time, the supply of thermal energy to the flow, as it occurs in the process 1-5, results in an increase of the internal energy of the gas, its temperature and entropy in comparison with the similar parameters of the gas during its isentropic expansion in the process 1-3.

The correlations between parameters of such adiabatic flows can be expressed by the equations

$$
\begin{aligned}
& p_{1} / p_{2}=\left(T_{1} / T_{2}\right)^{n /(n-1)}=\left(T_{1} / T_{3}\right)^{k /(k-1)}=\left(T_{1} / T_{5}\right)^{\gamma /(\gamma-1)}, \\
& \ln \left(p_{1} / p_{2}\right)=\frac{n}{n-1} \ln \left(\frac{T_{1}}{T_{2}}\right)=\frac{k}{k-1} \ln \left(\frac{T_{1}}{T_{3}}\right)=\frac{\gamma}{\gamma-1} \ln \left(\frac{T_{1}}{T_{5}}\right) \\
& \ln \left(p_{1} / p_{2}\right)=\frac{n}{n-k} \frac{\Delta s_{1-2}}{c_{v}}=\frac{\gamma}{k-\gamma} \frac{\Delta s_{1-5}}{c_{v}}
\end{aligned}
$$

which coincide with the similar ones for quasi-static polytropic processes with the polytropic exponents $n>k, k$ and $\gamma<k$ in the processes 1-2, 1-3 and 1-5 accordingly. Here, $\Delta s_{1-2}, \Delta s_{1-5}$ are the changes of entropy in the processes 1-2 and 1-5 correspondingly.

For an analysis of the adiabatic flows it is expedient to introduce a new concept of adiabatic heat capacities, in which a dependence of the system temperature not only on the thermal effects as usually but and on the mechanical ones could be taken into account too.

Depending on the form of energy in this case, it is possible here to specify the following:

- the heat capacity $c_{w}$ of kinetic influences at $s=$ const

$$
c_{w}=\omega \delta \omega / \partial T=c_{p}=k R /(k-1),
$$

- the heat capacity of mechanical influences $c_{l}$

$c_{l}=\delta l / \partial T$

- and the heat capacity $c_{n}$ of the polytropic processes caused by a combined influence of several energy factors

$c_{n}=\partial\left(\omega^{2} / 2+l\right) / \partial T=n R /(n-1)$.

One of such heat capacities, namely $c_{w}$, as is shown above, is already used in an implicit form in the quasi-static method.

The seeming heat capacity $c_{l}=\delta l / \partial T$ of the mechanical effects in the equation (38) characterizes a contribution of energy in the form of work in the total heat capacity $c_{n}$ of a gas flow, and its value can be found as a difference between the total heat capacity $c_{n}$ of a flow and its isobaric heat capacity $c_{p}=k R /(k-1)$

$$
\begin{aligned}
& c_{l}=\Delta c_{p}=c_{n}-c_{p}=n R /(n-1)-k R /(k-1)= \\
& =c_{v}(k-n) /(n-1)=c_{c}
\end{aligned} .
$$

In this event the heat capacities of mechanical effects in essence coincide with the similar heat capacities of the thermal effects $c_{c}=\delta q / \partial T$ in a quasi-static polytropic process

$c_{c}=c_{v}+R /(1-n)=c_{v}(n-k) /(n-1)$.

Moreover, such heat capacities can also be used for definition of entropy changes in the dynamic polytropic processes. Thus, for example, an entropy change of the isothermal process 4-2 in Fig. (1) can be represented in terms of both the pressure and the heat capacity:

$$
\begin{aligned}
& \Delta s_{2-4}=R \ln \left(p_{2} / p_{4}\right)=R\left[\ln \left(p_{2} / p_{1}\right)-\right. \\
& \left.-\ln \left(p_{4} / p_{1}\right)\right]=\Delta c_{p} \ln \left(T_{2} / T_{1}\right)
\end{aligned}
$$

The quantity of specific work $l$ necessary for a change in entropy of an adiabatic gas flow by the value $\Delta s_{1-2}=s_{2}-s_{1}$ can be represented through the expression

$$
\begin{aligned}
& l=T_{m} \Delta s=R T_{m} \ln \left(p_{2} / p_{4}\right)=T_{m}[k R /(k-1)- \\
& -n R /(n-1)] \ln \left(T_{2} / T_{1}\right)=\Delta c_{p} \Delta T
\end{aligned} .
$$

In Eq. (44) it is assumed that the processes 1-2 and 2-4 have got equal entropy changes $\Delta s_{1-2}=\Delta s_{2-4}$, $T_{m}=\left(T_{2}-T_{1}\right) / \ln \left(T_{2} / T_{1}\right)$ being the average temperature of the temperature range $\Delta T=\left(T_{2}-T_{1}\right)$ and $\Delta c_{p}=\left(c_{p}-c_{n}\right)=c_{l}$ being the difference of corresponding heat capacities in the isentropic and polytropic gas flows.

In so doing, the quantity of work consumed in the adiabatic dynamic process is equal to the quantity of heat released by the system in the similar polytropic quasi-static process.

The change of temperature in this case is defined by relationship (45)

$c_{p} \Delta T=-\Delta \omega^{2} / 2+c_{l} \Delta T$

and can be represented by using the heat capacities of both the thermal and mechanical influences

$\Delta T=l / c_{l}=\Delta h / c_{p}=(\Delta h-l) / c_{n}=\Delta(p v) / R$.

\subsection{The Polytropic Exponents of the Adiabatic Flows in Mechanic-Geometrical Nozzles}

Taking into account such heat capacities, the equation (24) representing the polytropic exponents of the adiabatic flows in the mechanic-geometrical nozzles formally coincides with the known definition of the polytropic exponents of quasi-static processes 
$n=-\frac{v d p}{p d v}=\frac{c_{w} \pm c_{l}}{c_{w} \pm c_{l}-R}=\frac{c_{p} \pm c_{l}}{c_{v} \pm c_{l}}$.

Besides, in this case the polytropic exponents can also be represented by other expressions

$n=-\frac{v d p}{p d v}=\frac{\Delta \omega^{2} / 2-l}{\Delta \omega^{2} / 2-l-R \Delta T}$,

$n=\frac{\omega^{2} d f /\left[\left(M^{2}-1\right) f\right]-d l}{\omega^{2} d f /\left[\left(M^{2}-1\right) f\right]-d l-R d T}$,

pointing to a possible dependence of these exponents on the Mach number, which was not considered earlier.

According to these equations, an adiabatic exponent of a gas flow in a mechanic-geometrical nozzle can be equal to the ratio $n=k=c_{p} / c_{v}$ only at its relatively moderate subsonic $\left(M^{2}<<1\right)$ velocities or at higher supersonic $\left(M^{2} \gg 1\right)$ ones.

However, as the gas velocity increases due to the narrowing of a nozzle channel and the application of external mechanical effects, its regime of flowing can deviate from the isentropic one and approach the isothermal one because at $M \rightarrow 1$ the polytropic exponent also tends to unit $n \rightarrow 1$.

In this case a change of flow enthalpy is equal to zero $\Delta h=c_{p} \Delta T=0$, and the equation of continuity $d G=d(\omega \rho f)$ can be represented in the form of expression

$\frac{d G}{G}-\frac{d f}{f}-\frac{d \rho}{\rho}=0$,

provided that the gas flow velocity in the critical outflow section of such a nozzle is equal to a sonic speed $\omega=a=$ const .

According to Eq. (50), the density of subsonic gas flow can increase both at raising the mass gas flow $d G>0$ and at decreasing its cross-sectional area $(d f<0)$.

Generally, the adiabatic exponents of the adiabatic gas flows can coincide with the similar polytropic exponents of the corresponding quasi-static processes. In particular, the ratios $n=k, c_{n}=\left(c_{\omega}+c_{l}\right)=c_{p}$ correspond to the isentropic gas flows. An isochoric regime of gas flowing corresponds to the other conditions, at which $n \rightarrow \pm \infty, c_{n} \rightarrow R$, the work necessary for such a regime can be represented by expression

$\delta l_{t}=c_{l} d T=\left[c_{v}(n-k) /(n-1)\right] d T=c_{v} d T$.

The Bernoulli equation in this case is transformed to a view being characteristic for a flow of an incompressible fluid, i.e. for the flow with constant density $\rho=$ const

$\Delta p / \rho+l=-\Delta \omega^{2} / 2$.

Diagrams (trajectories) of such dynamic adiabatic processes in various coordinate systems can coincide with similar trajectories of the corresponding quasi-static polytropic processes, as shown in Fig. (2) where the isochoric process 1-2 with the polytropic exponent $n \rightarrow \pm \infty$, the isentropic process 1-3 with the exponent $n=k$, as well as the polytropic process 1- 4 with $k>n>1$ can simultaneously be considered both as quasi-static polytropic and as dynamic adiabatic ones.

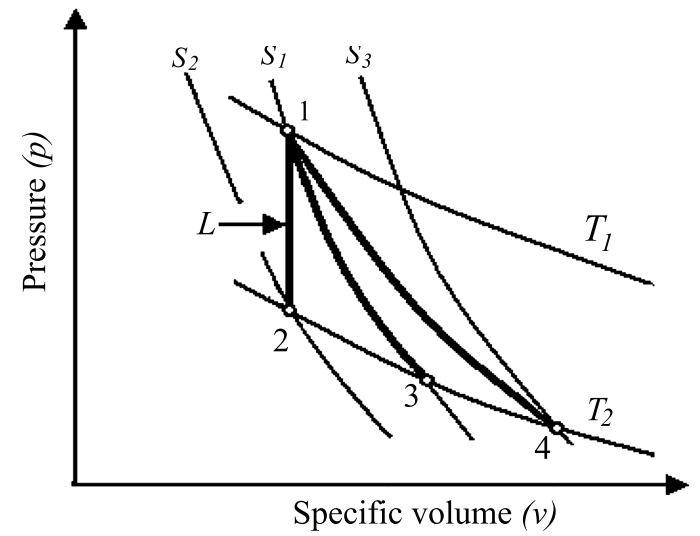

Fig. (2). The adiabatic diagrams of gas flows in an equal temperature interval under the following conditions: $\rho=1 / v=$ const in the process 1-2, $p v^{k}=$ const in the process 1-3, and $p v^{n}=$ const in the process $1-4$, where $s_{1}, s_{2}, s_{3}$ are isentropic curves.

\section{ADDITIONAL INTERPRETATION OF SOME NO- TIONS OF THE QUASI-STATIC METHOD}

The classical method of quasi-static equilibrium for the closed systems can be represented as a special case of the general thermodynamic model.

The basic assumptions of this method as to the exclusive connection of entropy with the heat exchange processes and the impossibility of the ideal gas adiabatic curves to intersect among themselves are not carried out strictly enough not only in the open systems but also in the closed statically equilibrium ones.

In particular, the adiabatic curves (trajectories) of ideal gases can intersect among themselves in any coordinate systems including $\mathrm{T}$ (temperature) - $\mathrm{s}$ (entropy) diagrams if they represent various ideal gases with different adiabatic indexes, for example $k_{1}>k_{2}>k_{3}$. In this case such adiabatic curves can coincide with the similar trajectories of polytropic processes if their adiabatic indexes coincide with the similar polytropic exponents $k_{i}=n_{i}$, as shown in Fig. (3).

The other idea of the quasi-static method as to the exclusive dependence of the thermodynamic cycle efficiency on the temperatures of thermal sources is not general enough also since it can be complemented by the other notion of dependence of such efficiency on the corresponding temperatures of working fluids.

Such a conclusion about the nature of the quasi-static equilibrium method can be made on the basis of an extended interpretation of the Carnot cycle for ideal gas, the diagram 

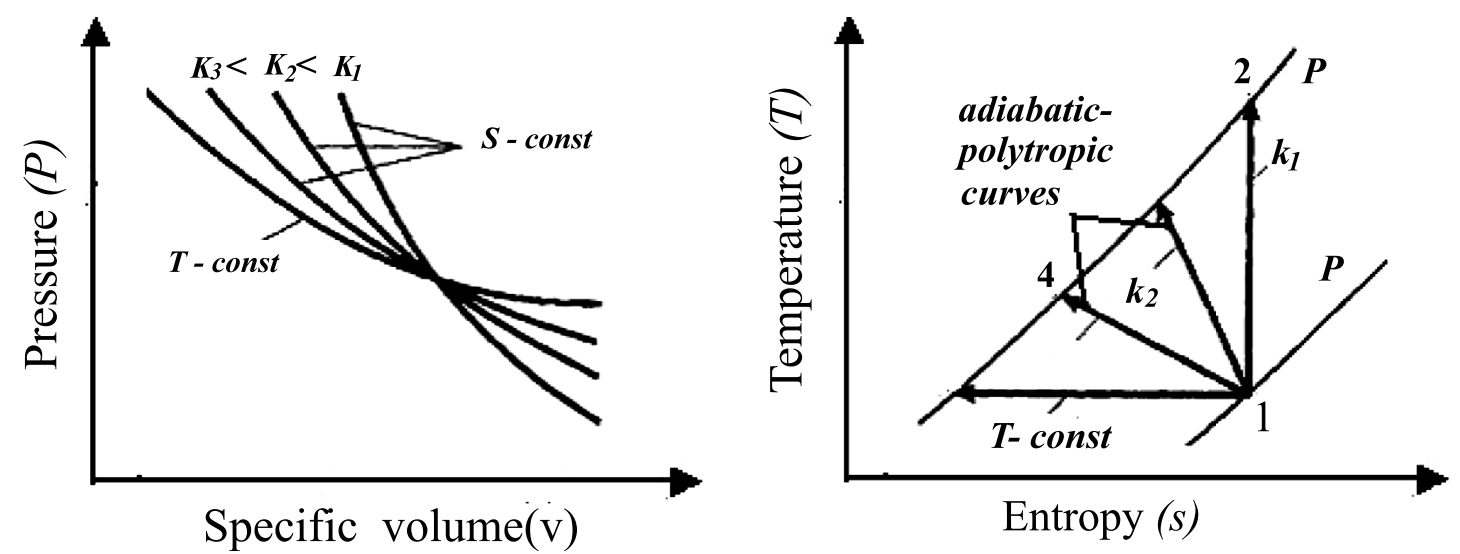

Fig. (3). The adiabatic and polytropic quasi-static curves of three various ideal gases with adiabatic $k_{1}, k_{2}, k_{3}$ and polytropic $n_{1}, n_{2}, n_{3}$ indexes under the condition that $k_{i}=n_{i}$.

of which is represented by the processes $1 *_{-}-2 *_{-} 3 *_{-} 4 *$ in Fig. (4).

According to such an interpretation the isothermal processes of this cycle ( $T=$ const $)$ can also be considered as the isoenergetic processes in which internal energy of a gas is kept as a constant ( $U=$ const $)$.

In this case the isothermal processes $\left(1 *_{-} *^{*}\right)$ and $\left(3 *_{-} 4^{*}\right)$ of this cycle along with the flows of heat energy $q$ have also the equivalent flows of mechanical energy, work $l$, which are usually not considered. When taking it into account, both the respective entropy changes $\Delta s$ in these processes and cycle efficiency $\eta$ can be defined not only by the known expressions

$\Delta s=q_{i} / T_{i}^{*}$

$\eta=\left(q_{1}-q_{2}\right) / q_{1}=1-T_{2}^{*} / T_{1}^{*}$,

in which $T_{i}^{*}$ are the temperatures of heat sources, but also by other expressions

$\Delta s=l_{i} / T_{i}^{*}$,

$\eta=\left(l_{1}-l_{2}\right) / l_{1}=1-T_{2}^{*} / T_{1}^{*}$,

in which $T_{i}^{*}$ are the temperatures of a working fluid but not temperatures of thermal or mechanical energy sources.

Moreover, the well-known constancy of entropy or the equality $\Delta s=0$ in the adiabatic processes $(2 *-3 *)$ and $\left(4^{*}-1 *\right)$ of this cycle in which $q$ is equal 0 can be explained not only by absence of the heat exchange processes, as it is usually supposed, but also by the mutual compensation of two component parts of the entropy change $\Delta s=\Delta s(T)+\Delta s(v)$, one of them $\Delta s(T)$ is defined by a change in gas temperature and another $\Delta s(v)$ is defined by a change in gas volume or by gas density $\rho$.

The similar results that allow the entropic changes to be defined by means of the work quantity and the temperatures of the working fluid (instead of the temperatures of the heat sources) have also been obtained with reference to the Lorentz cycle (Fig. 4) including its polytropic processes represented by the equation $p v^{n}=T v^{n-1}=$ const .

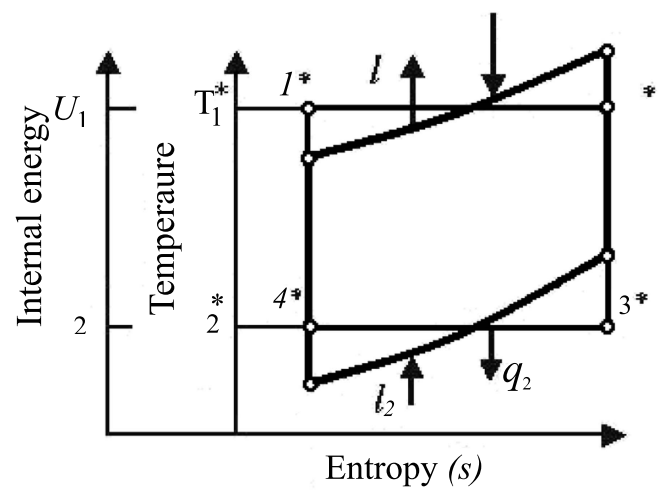

Fig. (4). The Carnot $\left(1^{*}-2^{*}-3^{*}-4^{*}\right)$ and Lorentz $(1-2-3-4)$ cycles with the quasi-static processes.

In particular, in this case both the entropy changes in the polytropic processes of such cycles and their efficiency can be expressed not only by the traditional equations

$$
\begin{aligned}
& \Delta s=c_{v}[(n-k) /(n-1)] \ln \left(T_{2} / T_{1}\right), \\
& \eta=1-T_{2}^{*} / T_{1}^{*},
\end{aligned}
$$

which include the initial $T_{1}$, final $T_{2}$, and middle $T_{i}^{*}$ temperatures of thermal sources, but also by using the additional expressions

$$
\begin{aligned}
& \Delta s_{1-2}=c_{v}(n-k) \ln \left(v_{1} / v_{2}\right)=R \ln \left(v_{1}^{*} / v_{2}^{*}\right), \\
& \eta=\left(l_{1}^{*}-l_{2}^{*}\right) / l_{1}^{*}=1-T_{2}^{*} / T_{1}^{*},
\end{aligned}
$$

taking into account the mechanical interactions $l_{1}^{*}, l_{2}^{*}$ and the middle temperatures of a working fluid $T_{1}^{*}$ and $T_{2}^{*}$ at the upper and lower temperature levels of the cycle accordingly, where $T_{i}^{*}=\left(T_{i+1}-T_{i}\right) \ln \left(T_{i+1} / T_{i}\right)$. 
Moreover, the temperature range $\Delta T=\left(T_{1}^{*}-T_{2}^{*}\right)$ of such a cycle as well as its efficiency $\eta$ depends on the properties of the working fluids, including such characteristics as the polytropic exponent $n$, the thermal capacities $c_{v}^{*}$ and $c_{n}{ }_{n}$ of the working fluid in such processes

$n=-v d p / p d v=c_{n}^{*} / c_{v}^{*}=\left(c_{p}-c\right) /\left(c_{v}-c\right)$,

$c_{v}^{*}=p \partial v / \partial T=\left(c_{v}-c\right)=R /(n-1)$,

$c_{n}^{*}=v \partial p / \partial T=\left(c_{p}-c\right)=n R /(n-1)$.

These thermal capacities in essence are similar to the known thermal capacities $c_{p}$ (at $p=$ const) and $c_{v}$ (at $v=$ const ) and differ from them by the size of corrections $c=\partial q / \partial T$ that consider the influence of internal or external energy exchange and of changes in structure or composition of a working fluid.

With reference to the open dynamic systems the entropy can be considered not only as a function of thermal interactions $d s=\delta q / T$, as usually, but also of the mechanical ones $d s=\delta l / T$.

Besides, the entropy changes can be represented in this case as a function of the molecule concentrations (or the thermal capacities) of a system in the isothermal processes for which the external heat exchange is not necessary:

$$
\begin{aligned}
& \Delta s=k N_{1} \ln \left(v_{1} / v_{2}\right)=k N_{1} \ln \left(\rho_{2} / \rho_{1}\right)= \\
& =k N_{1} \ln \left(N_{2} / N_{1}\right)=k \Delta N=\Delta R_{v}
\end{aligned} .
$$

Here, $N_{1}, N_{2}$ are the initial and final concentrations of molecules, $\Delta N=\left(N_{2}-N_{1}\right)<<N_{1}$ is a change of the molecule concentration, and $\Delta R_{v}=R_{v} \ln \left(R_{v 2} / R_{v 1}\right)=k \Delta N$ is a change of the gas constant $R_{v}$ related to the units of the volume but not of the mass as usually.

\section{PECULIARITIES OF THE APPLICATION OF THE $2^{\text {ND }}$ LAW OF THERMODYNAMICS TO THE OPEN NON-EQUILIBRIUM SYSTEMS}

The used approach corresponds to the extended interpretation of the second law of thermodynamics according to which the efficiency $\eta$ of a cycle is determined by the relative temperature difference of a working fluid (but not by that of the heat sources) at the upper and lower temperature levels of the cycle $[4,5]$.

Such an interpretation of this law permits the regenerative cycles that have got a contact only with one thermal source to exist.

One of the ways of the realization of such an opportunity is creation of the cycles with dynamic regeneration at which the energy of a cycle closure arising at its lower temperature level will be transformed not into the thermal energy as in the Carnot cycle but into the kinetic energy of the flow.
The performance $\eta$ of such regenerative closed cycles with one thermal source can coincide with the similar expression for the Carnot cycle

$\eta=1-T_{2}^{*} / T_{1}^{*}$

However, unlike the Carnot cycle, $T_{2}^{*}$ and $T_{1}^{*}$ are here suitable average temperatures of a working fluid (but not of those of the thermal sources as it is usually supposed), and the quantity $\eta$ characterizes the ratio between the useful and full work of a cycle.

As an example, the diagram of the closed ideal gas cycle with dynamic regeneration is represented in Fig. (5). This cycle consists of the isothermal process 2-3 with the flows of the heat $q_{1}$ and the work $l_{l}$, and of the three adiabatic processes with the adiabatic indexes being equal to $n=k=c_{p} / c_{v}$ in the processes 1-2 and 3-4, as well to $n \rightarrow \infty$ in the process $4-1$.

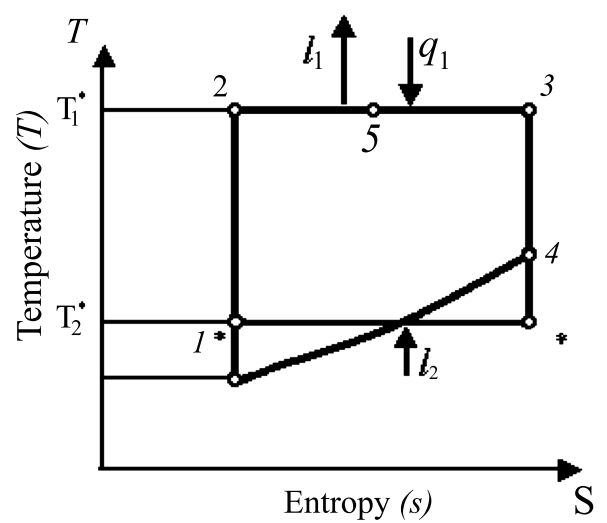

Fig. (5). $T-S$ diagram of the closed ideal gas cycle with dynamic regeneration.

During process 4-1 the adiabatic gas flow is partially compressed with the transformation of the consumed mechanical energy into the kinetic energy which, in turn, regenerates in the form of thermal energy at the upper temperature level $T_{1}^{*}$ of the cycle. The full work of the cycle amounts to $l_{1}=l^{*}+l_{2}=q_{1}+l_{2}=T_{1}^{*} \Delta s$, its work output $l^{*}$ is equal to $q_{1}\left(l^{*}=q_{1}\right)$, while the work of the regeneration in the process 2-5 is equal to $l_{2}=T_{2}^{*} \Delta s=\Delta \omega^{2} / 2$.

Moreover, the similar approach can also be realized in the cycles with the use of the vapor as a working fluid. One of such opportunities is a new design of absorption combined heat pump (CHP) offered by the author [9]. It is intended for both joint and separate production of electricity, heat and cold from different types of fuel, including renewable low-potential energy sources. The schematic diagram of the offered CHP is represented in Fig. (6).

In such a CHP, unlike the similar machines known earlier, the absorption of vapor in a weak solution (brine) can be an adiabatic process, and the lower border of its useful temperature range can be reduced to the values smaller than the temperature level of an environment. During the absorption 


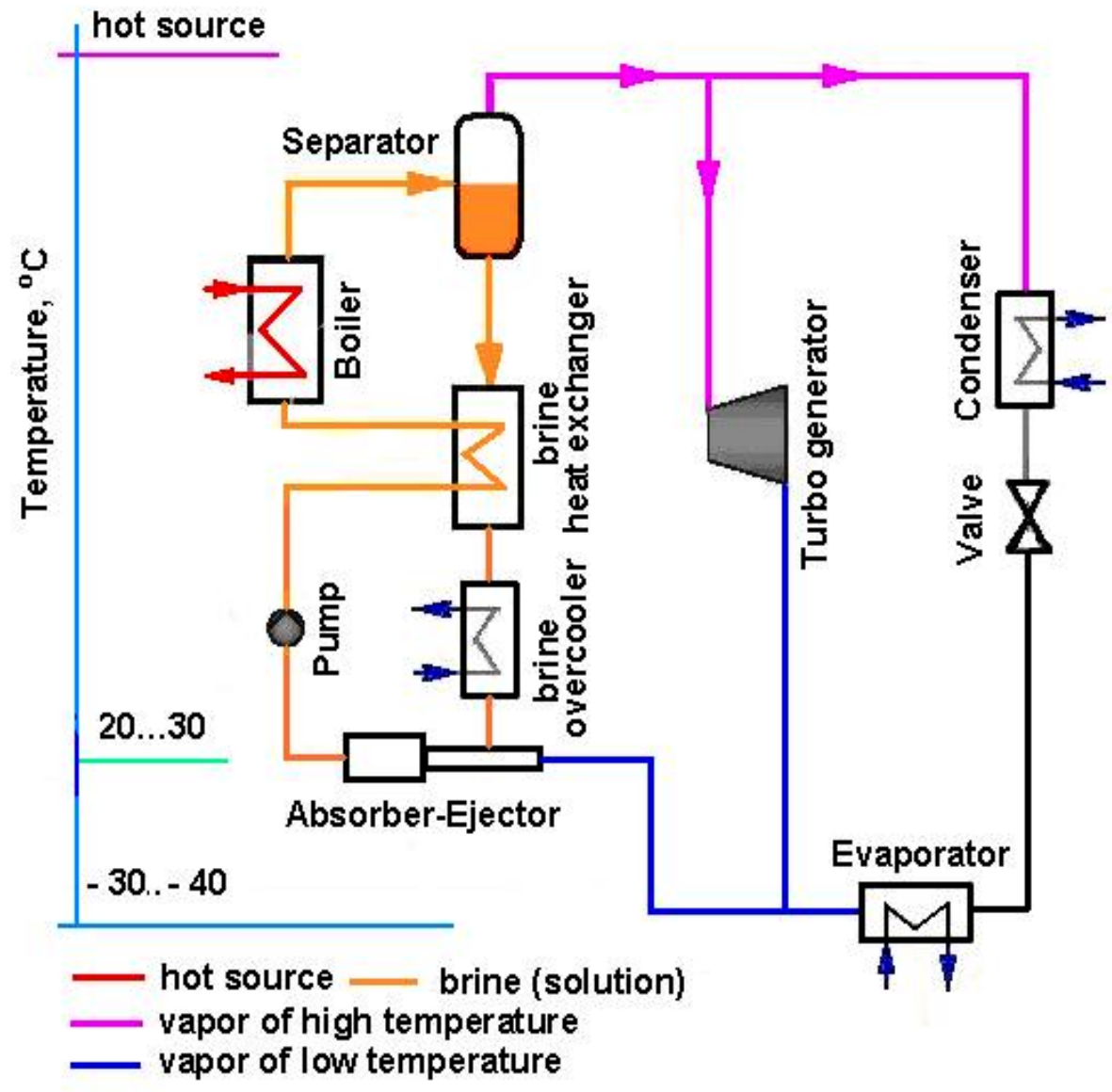

Fig. (6). The CHP schematic diagram.

of such a CHP, the temperature of the liquid solution (of the absorbent) increases due to the thermal energy that is released in this process, and in doing so the solution pressure also grows by means of an ejector in which a difference of potential energies of the solution in the separator and the absorber is used.

The offered CHP allows the ecological safety and economic efficiency to be significantly increased (almost by a factor of 1.5-2) in comparison with any similar systems known earlier. This technology can be basically realized with the commercially available equipment, so it can be implemented industrially in a relatively short time.

\section{CONCLUSION}

The analysis of the statically non-equilibrium systems carried out with the application of the method of dynamic equilibrium enables the thermodynamic concept to be extended beyond the borders of the classical method based on the notions as to the equilibrium systems and their quasistatic processes.

Within the framework of such a concept, the differences of energy potentials between a system and an environment are taken into account as additional components of the equations of the first law and the equations of system state.

In this case, the entropy is the function of the system parameters, in which the known inseparable association of the entropy with the heat exchange processes is not necessary and its change in the adiabatic processes can be not only a positive quantity as it takes place in the quasi-static processes but also a negative quantity.

The adiabatic processes running with entropy decreasing can be particularly realized in the flows of working fluids, for example in the gas flows with the fixed density, and the combination of the Poisson's adiabatic processes with the Bernoulli's similar processes creates an opportunity to regulate the adiabatic indexes (parameters of adiabatic curves) similar to the adjusting of the polytropic exponents in the quasi-static processes.

At such an approach the second law of thermodynamics has the extended interpretation according to which the performance of heat-mechanic transformations in the cyclic processes is defined by the relative temperatures difference of a working fluid at the upper and lower temperature levels of a cycle, but it is not necessarily by the analogous temperatures of thermal sources as it was supposed earlier.

Such an interpretation unlike classical one corresponds to the equivalence principle, allows considering behavior features of non-equilibrium irreversible systems as well, and supposes an opportunity for existence of the thermodynamic cycles having thermal contact only with one thermal reservoir.

The obtained results also give grounds to believe that the new generation of thermal machines capable to provide sub- 
stantial increase of the production efficiency of electricity, heat, and cold in using both renewable energy sources and fossil fuel can be created now even at the existing level of techniques.

The use of these possibilities can apparently be considered as the relatively simple and economical method for the solution of some modern topical problems of the environmental and energy safety.

\section{REFERENCES}

[I] Samkhan, I. In ENERGEX 2000, Proceedings of the 8th International Energy Forum, Las Vegas, USA, July 23-28, 2000, pp. 865870 .

[2] Leontovich, M. A. Introduction in Thermodynamics. Statistical Physics; State Publishing House "Nauka": Moscow, 1983.

[3] Samkhan, I. On the relationship between the specific heat and entropy of thermodynamic systems. Phys. Dokl., 1996, 1, 16-18.
[4] Samkhan, I. New possibilities for energy production from renewable low-potential sources. Appl. Energy, 2003, 1-2, 203-209.

[5] Samkhan, I. On the relationship between static and dynamic models of thermodynamics. Dokl. Phys., 2006, 12, 647-650.

[6] Samkhan, I. In 2004 Purdue Conferences, Proceedings from the Seventeenth International Compressor Engineering Conference at Purdue and the Tenth International Refrigeration and Air Conditioning Conference at Purdue, Purdue University, Lafayette, USA, July 12-15, 2004. www.adpc.purdue.edu/physfac/prnt/Welcom.html

[7] Samkhan, I. In Mathematical Methods in Techniques and Technologies, Proceedings of the 20th International Scientific Conference, Yaroslavl, Russia, 2007, Vol. 3, pp. 10-12.

[8] Kruglov, M.; Mednov, A. Gas Dynamics of the Combined InternalCombustion Engines; Machinery: Moscow, 1988.

[9] Samkhan, I. Method and Device for Converting Thermal Energy into Electricity, High-Potential Heat and Cold. International Application No.: PCT/RU2007/000238, Pub. No.: WO/2008/143542, November 27, 2008

(C) Igor I. Samkhan; Licensee Bentham Open.

This is an open access article licensed under the terms of the Creative Commons Attribution Non-Commercial License (http://creativecommons.org/licenses/bync/3.0/) which permits unrestricted, non-commercial use, distribution and reproduction in any medium, provided the work is properly cited. 